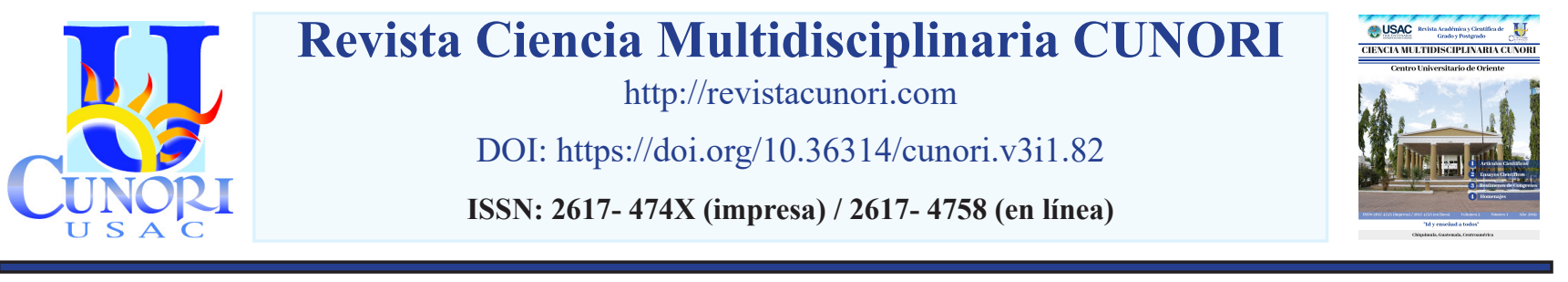

Como citar el artículo

Díaz, J., Icaza, G., Arriola, C., Mazariegos, E., (2019). Recurrencia de hernias inguinales en el Hospital de Zacapa. Revista Ciencia Multidisciplinaria CUNORI, 3(1), 75-84. DOI: https://doi.org/10.36314/cunori.v3i1.82

\title{
Recurrencia de las hernias inguinales en el hospital regional de Zacapa
}

\section{Recurrence of inguinal hernias in the Zacapa regional hospital}

\author{
Juliana Díaz*, Graciela Icaza, Carlos Arriola, Edvin Mazariegos \\ Centro Universitario de Oriente (CUNORI), Universidad de San Carlos de Guatemala \\ Recibido: 05 de agosto de 2018 / Revisión: 20 de septiembre de 2018 / Aceptado: 19 de enero de 2019 \\ Disponible en internet el 30 de agosto de 2019

$\begin{array}{ll} & * \text { Autor para correspondencia. } \\ \text { Resumen } & \text { Correo electrónico: juliana_007diaz@hotmail.com }\end{array}$

Q1 trauma craneoencefálico (TCE) es una lesión del tejido cerebral causada por un traumatismo directo o indirecto a nivel craEneal, clasificándose según su gravedad utilizando la escala de coma Glasgow en leve, moderado y severo. Dicha patología es una importante causa de muerte en la población joven, por lo que se realizó este estudio en el Hospital Nacional de El Progreso por la falta de recursos para el manejo clínico, estabilización y rehabilitación del paciente, por lo tanto se hace importante caracterizar al paciente que sufrió trauma craneoencefálico por cualquier causa; realizándose un estudio descriptivo retrospectivo revisando 156 expedientes con historia de trauma craneoencefálico. Los datos fueron recopilados en una boleta de recolección. Del total de casos el género más afectado fue el masculino 84\% (131), la edad más frecuente se ubicó de 21 a 30 años 28\% (43), la causa más frecuente fue el accidente de tránsito 58\% (91), el TCE leve fue el más común 81\% (97), solo un 20\% (31) se realizó TAC, la lesión mayormente observada por tomografía fue el edema cerebral 39\% (12), 5\% (7) de los pacientes tuvieron complicaciones infecciosas, 6\% (8) requirieron ventilación asistida, la tasa de mortalidad que se obtuvo fue del 3\% (5) presentando muerte al arribo. Por lo anterior se sugiere concientizar a la población en educación vial y medidas de seguridad para pilotos y peatones, y así modificar conductas de riesgo para evitar el aumento de la mortalidad por esta patología.

Palabras clave: trauma craneoencefálico, caracterización, escala de Glasgow, lesión cerebral

Abstract

Cranioencephalic trauma (CET) is a brain tissue injury caused by direct or indirect cranial trauma, classified according to severity using the Glasgow coma scale in mild, moderate and severe. This pathology is an important cause of death in the young population, so this study was conducted in the National Hospital of El Progreso for lack of resources for clinical management, stabilization and rehabilitation of the patient, therefore it is important to characterize the patient who suffered cranioencephalic trauma from any cause; A retrospective descriptive study was conducted using 156 records with a history of cranioencephalic trauma. The data was collected on a data collection ticket to be digitized in Microsoft Excel 2010 and then analyzed according to the results obtained. Of all the files, the most affected gender was male $84 \%$ (131), the most frequent age was from 21 to 30 years $28 \%$ (43), the most frequent cause was traffic accident 58\% (91), mild TBI was the most common 81\% (97), only 32\% (31) TAC was performed, the injury mostly observed by tomography was cerebral edema 39\% (12), 5\% (7) of patients had infectious complications, $6 \%$ (8) required assisted ventilation, the mortality rate was 3\% (5) presenting death upon arrival. Therefore, it is recommended to raise awareness among the population in road safety education and safety measures for pilots and pedestrians, and thus modify risk behaviors to avoid an increase in mortality due to this pathology.

Keywords: inguinal hernia, recurrence of inguinal hernia, surgical technique 


\section{Introducción}

La corrección de hernias inguinales es un procedimiento quirúrgico que se realiza con más frecuencia en los servicios de cirugía general. De acuerdo con datos del National Center for Health Stadistics, la hernioplastia encabeza la lista de las cinco operaciones quirúrgicas mayores que se han realizado durante varios años (Chinchilla, 2007). Independientemente del número exacto de hernioplastias realizadas, es evidente que constituye un problema de gran magnitud, sobre todo las recurrencias. Cuando la hernia experimenta recurrencia meses o años más tarde, es difícil atribuir el problema a un error quirúrgico. Se ha visto que la recurrencia de la hernia inguinal según la OMS va del 1 al 17\% más frecuente después de hernioplastia primaria de Bassini la cual va del $6 \%$ al $7.3 \%$, siendo más confiable la técnica de Shouldice con $0.6-2.6 \%$ de recurrencias (Barrera, 2015).

La gran diversidad de posibilidades que hay para el manejo de la hernia inguinal, hace que la escogencia de un tipo específico de procedimiento quirúrgico sea muy variable y esté ligado más a una preferencia personal y no a criterios relacionados con el paciente y a los mejores resultados posibles (Palacio, 2016). Una de las técnicas quirúrgicas que ha venido fortaleciéndose y demostrando mejores indicadores de recuperación en el paciente, ha sido la asociada al uso de malla con una técnica libre de tensión. A la luz de los conocimientos actuales, mediante múltiples estudios y metaanálisis se puede afirmar claramente que el uso de malla con técnicas específicas como la de Lichtenstein ha reducido significativamente la tasa de recurrencia de estas hernias en, aproximadamente, 50 a $75 \%$; además, muestran disminución de complicaciones como la recurrencia (Buitrago, Vera, Martínez \& Muñoz, 2014). El estudio trata de determinar la recurrencia de hernias inguinales y las técnicas de corrección quirúrgica en 137 pacientes mayores de 18 años en los servicios de cirugía general de adultos del Hospital Regional de Zacapa durante periodo de enero 2013 a diciembre de 2017.

\section{Materiales y métodos}

Estudio descriptivo - retrospectivo. Se revisaron los 137 (100 \%) expedientes clínicos de los pacientes que acudieron al Hospital Regional de Zacapa y fueron ingresados a los servicios de cirugía general de adultos con diagnóstico de hernia inguinal, durante enero de 2013 a diciembre de 2017. Se incluyeron los expedientes de hombres y mujeres mayores de 18 años con datos de recurrencia de hernia inguinal.

Se recolectaron los datos con base a la boleta diseñada específicamente para el estudio. La boleta se divide por tres series, la primera constó de datos generales del paciente como número de boleta, número de registro del expediente clínico, año, edad, género y procedencia, segunda serie incluyó los tipos de hernias inguinal, localización anatómica y tipo de cirugía; tercera serie incluyó técnica quirúrgica y dispositivos utilizados en la segunda y tercera intervención como su tiempo de evolución en hernias inguinales recurrentes. Se tabularon los datos, se procedió a realizar las tabulaciones, la evaluación estadística y la realización del análisis correspondiente. 


\section{Resultados}

Género de pacientes con recurrencia de hernia inguinal

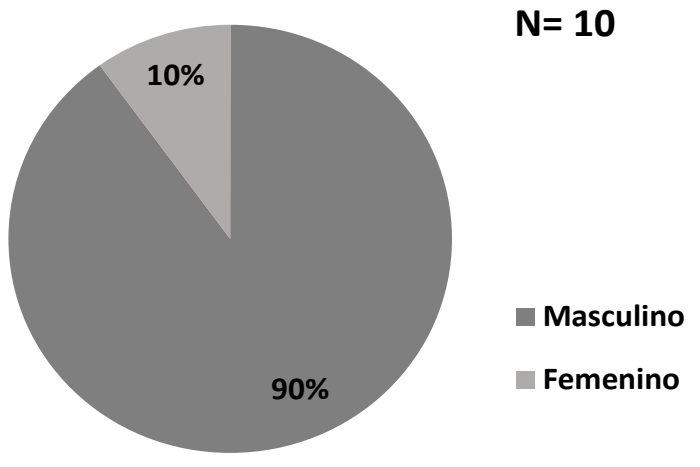

Figura 1. Distribución de pacientes por género con recurrencia de hernia inguinal atendidos en el servicio de cirugía general de adultos del Hospital Regional de Zacapa durante enero 2013 a diciembre de 2017.

El total de la muestra fueron 10 pacientes que presentaron hernia inguinal recurrente del cual el $90 \%$ (9) fueron del sexo masculino y el 10\% (1) corresponde al sexo femenino.

Edad de los pacientes con recurrencia de hernias inguinales

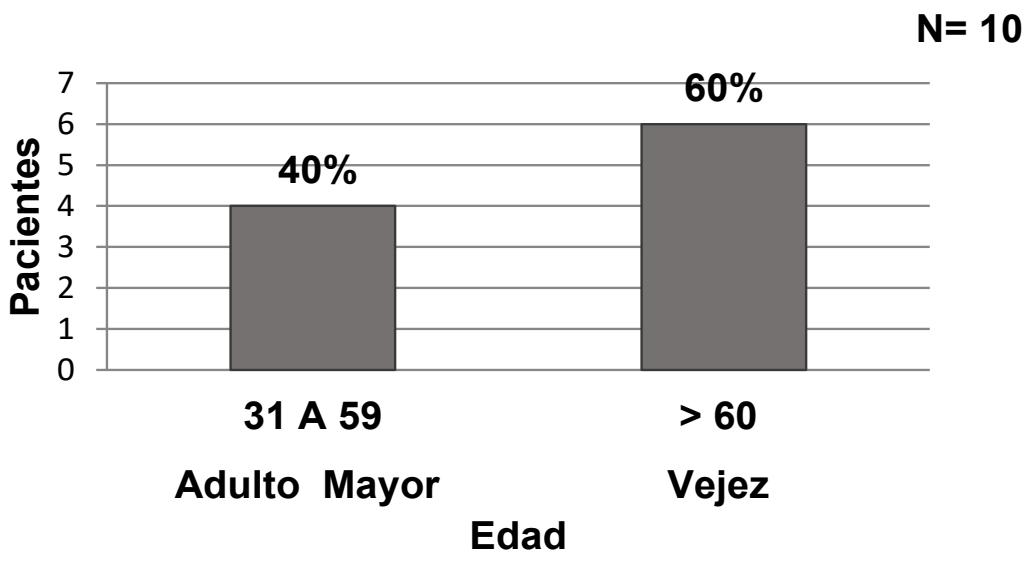

Figura 2. Distribución de pacientes por edad con recurrencia de hernias inguinales atendidas en el servicio de cirugía general de adultos del Hospital Regional de Zacapa durante enero de 2013 a diciembre 2017.

En la gráfica se aprecia que el rango por edades fue de la siguiente manera: 60\% (6) de los pacientes se encuentran en el rango > 60 años que corresponde a la categoría de vejez según la Organización Mundial de la Salud y el 40\% (4) en el rango de 31 a 59 años que corresponde a adulto mayor. 
Prevalencia de la recurrencia de hernias inguinales

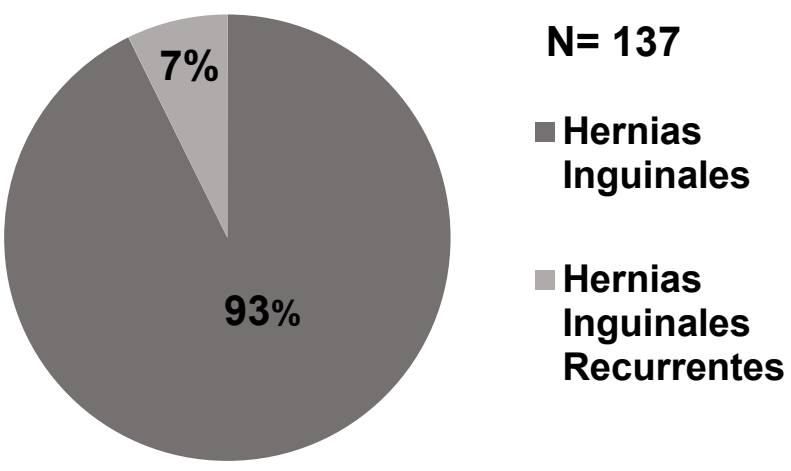

Figura 3. Distribución de la prevalencia de la recurrencia de hernias inguinales atendidas en el servicio de cirugía general de adultos del Hospital Regional de Zacapa durante enero de 2013 a diciembre 2017.

Según la gráfica 137 pacientes que fueron atendidos se encontró que el 93\% (127) casos fueron para tratamiento de hernia inguinal y según la Organización Mundial de Salud va de 1 a 17\% la tasa de recurrencia por lo tanto se determinó una prevalencia de 7\% (10) casos con hernia inguinal recurrente.

Cronología de la recurrencia de hernias inguinales

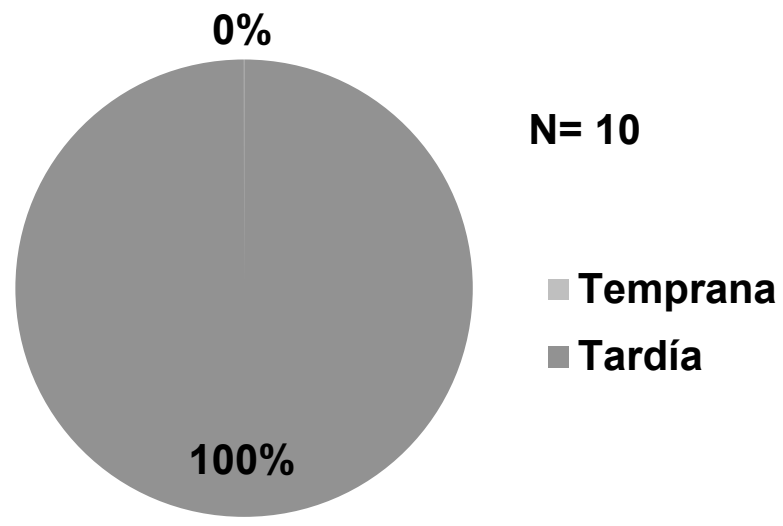

Figura 4. Distribución de la cronología de la recurrencia de hernias inguinales de pacientes atendidos en el servicio de cirugía general de adultos del Hospital Regional de Zacapa durante enero 2013 a diciembre 2017

La cronología de hernias inguinales recurrente el 100\% (10) son tardías y ningún caso de recurrencia temprana. 


\section{Recurrencia de hernia inguinal según escala de Campanelli}

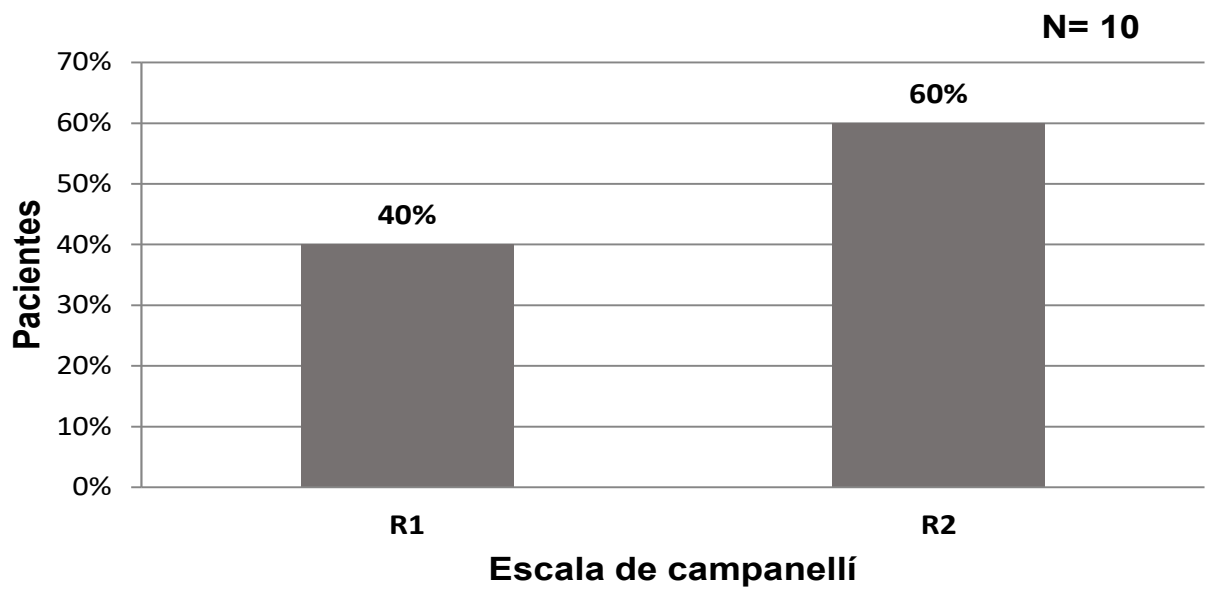

Figura 5. Distribución de la recurrencia de hernias inguinales según escala de Campanelli en pacientes atendidos en el servicio de cirugía general de adultos del Hospital Regional de Zacapa durante enero 2013 a diciembre 2017.

En la gráfica se aprecia que según escala de Campanelli $60 \%$ (6) de pacientes pertenecen a primera recidiva de hernia inguinal directa, seguida de $40 \%$ (4) de pacientes correspondientes a primera recidiva de hernia inguinal indirecta y sin ningún caso pertenecientes a todas las hernias inguinales recidivadas más de una vez.

Clasificación de la hernia inguinal recurrente según escala de Nyhus

$\mathrm{N}=10$

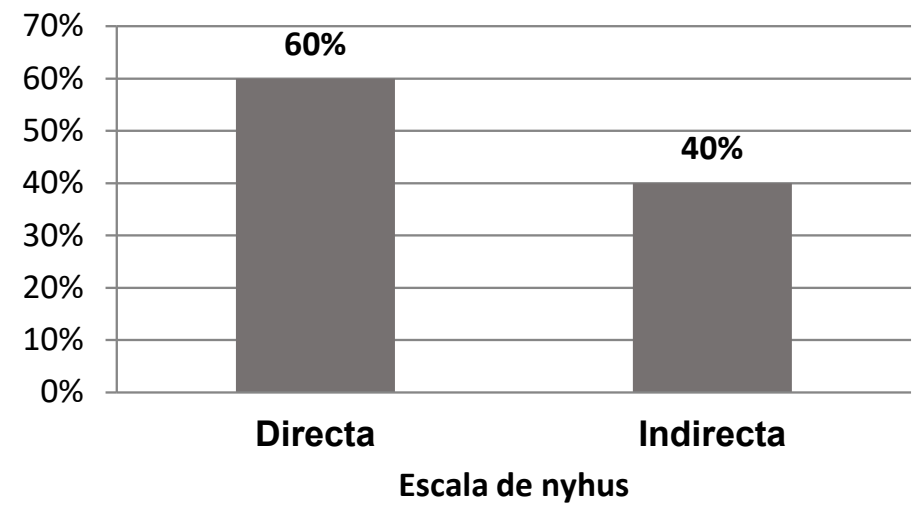

Figura 6. Distribución de la clasificación de la hernia inguinal recurrente según Escala de Nyhus en pacientes atendidos en el servicio de Cirugía General de adultos del Hospital Regional de Zacapa durante enero 2013 a diciembre 2017.

En la gráfica se aprecia la clasificación según la escala de Nyhus el 60\% (6) de los pacientes tuvo hernia inguinal recurrente tipo directa y el $40 \%$ (4) de los pacientes corresponde a hernia inguinal recurrente tipo indirecta. 
Localización anatómica de la hernia inguinal recurrente

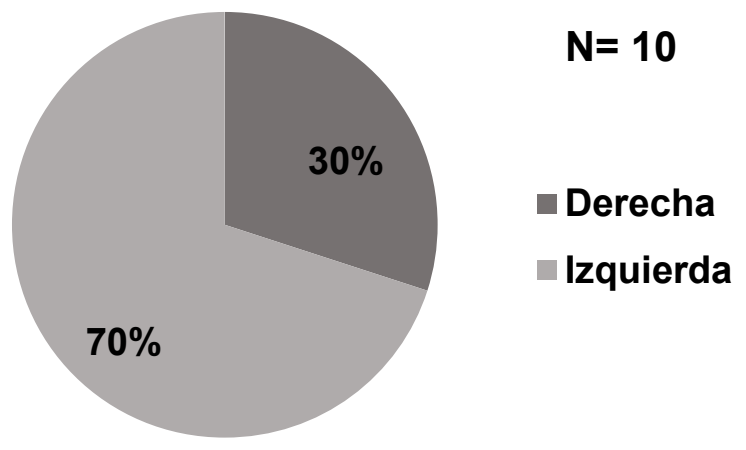

Figura 7. Distribución de la localización anatómica de la hernia inguinal recurrente en pacientes atendidos en el servicio de cirugía general de adultos del Hospital Regional de Zacapa durante enero 2013 a diciembre 2017.

Según la siguiente distribución referente a la localización anatómica de 10 pacientes el 70 \% (7) desarrollo hernia inguinal recurrente en lado de izquierda y el 30\% (3) de los pacientes presentó en lado derecho.

Técnica quirúrgica más utilizada en hernias inguinales

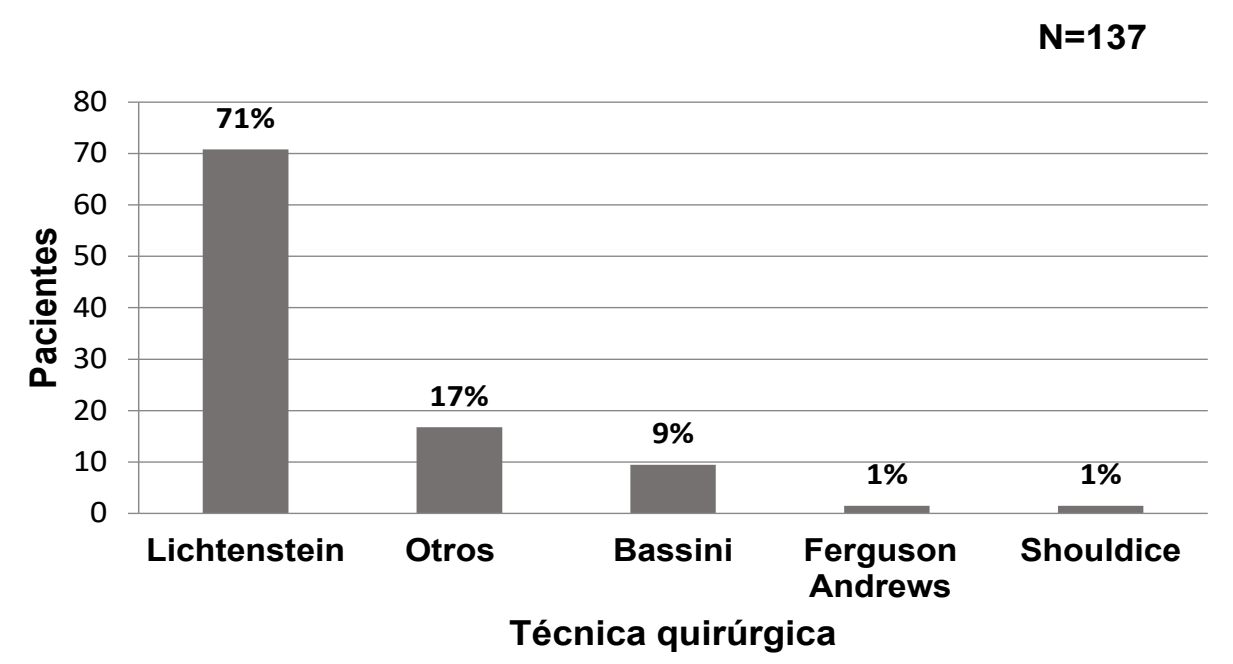

Figura 8. Distribución de la técnica más utilizada en la primera intervención quirúrgica de las hernias inguinales en pacientes atendidos en el servicio de cirugía general de adultos del Hospital Regional de Zacapa durante enero 2013 a diciembre 2017.

Según la gráfica se aprecia la técnica más utilizada en la primera intervención quirúrgica de137 pacientes con hernia inguinal; es la técnica Lichtenstein en 71\% (97) pacientes, y las menos utilizadas con $17 \%$ (23) casos con otros tipos de técnicas, 9\%(13) casos con técnica de Bassini, 1\% (2) con técnica de Ferguson Andrews y el 1\% (2) con técnica de Shouldice. 
Técnica quirúrgica más utilizada en hernias inguinales recurrentes

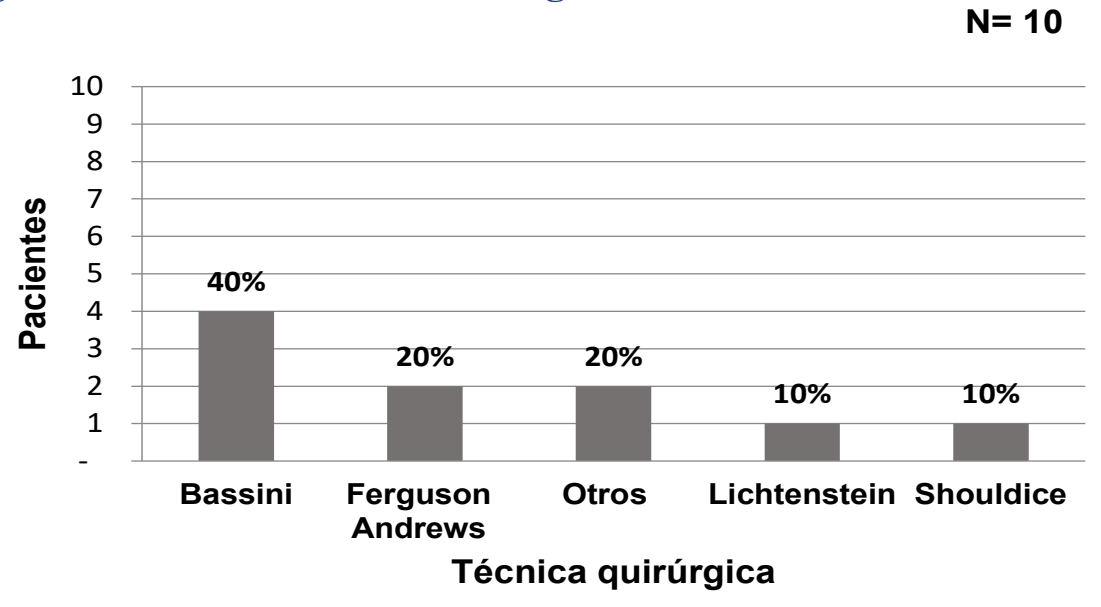

Figura 9. Distribución de la técnica más utilizada en la intervención quirúrgica primaria de hernia inguinal recurrente en pacientes atendidos en el servicio de cirugía general de adultos del Hospital Regional de Zacapa durante enero 2013 a diciembre 2017.

De acuerdo a la siguiente distribución de la técnica más utilizada en la intervención quirúrgica primaria del total de pacientes atendidos con hernia inguinal recurrente que corresponde a 10 pacientes; $40 \%$ (4) pacientes se realizó reparación con técnica de Bassini, en segundo lugar, el 20\% (2) casos con técnica de Ferguson Andrews, tercer lugar 20\% (2) casos con otras técnicas, en cuarto lugar 10\% (1) caso con técnica de Lichtenstein y 10\% (1) caso con técnica Shouldice.

Tipo de cirugía en las hernias inguinales recurrentes

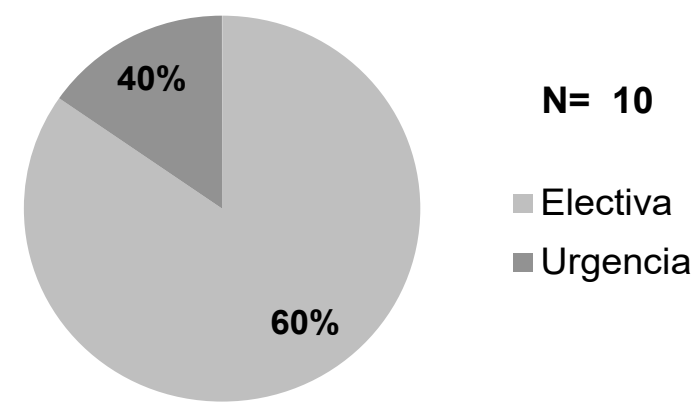

Figura 10. Distribución del tipo de cirugía en la hernia inguinal recurrente en pacientes atendidos en el servicio de cirugía general de adultos del Hospital Regional de Zacapa durante enero 2013 a diciembre 2017.

En la siguiente distribución se representa el tipo de cirugía al que fueron sometidos los pacientes con hernia inguinal recurrente que corresponde el $60 \%$ (6) fueron de forma electiva y el $40 \%$ (4) fueron realizadas de urgencia. 


\section{Discusión}

El presente estudio fue realizado con el propósito de determinar la recurrencia de hernia inguinal, en pacientes mayores de 18 años que fueron atendidos en los servicios de cirugía general de adultos del Hospital Zacapa durante enero de 2013 a diciembre de 2017. Se encontró que, de 137 casos que recibieron tratamiento quirúrgico de hernioplastia y/o herniorrafia, se obtuvo una prevalencia total del 7\% (10) casos que tuvieron recurrencia, quedando aun dentro de los rangos que la Organización Mundial de la Salud tiene para la recurrencia hernia inguinal que van del 1 al 17\%. Demostrando que el predominio de la recurrencia de hernia inguinal fue el sexo masculino con un total $90 \%$ de casos.

Del total de pacientes incluidos en el estudio, la distribución del rango por edades fue de la siguiente manera: con el 60\% (6) de los pacientes se encuentran en el rango mayor de 60 años, correspondiente según la Organización Mundial de la Salud a categoría de vejez y con el 40\% (4) en el rango de 31 a 59 años, correspondiente a la categoría de adulto mayor, sin ningún caso encontrado entre las edades de adolescencia tardía y adulto joven. En cuanto a la cronología de la recurrencia de hernia inguinal tiene predominio en los casos estudiados un 100\% (10) para recurrencia tardía, que equivale a una aparición después de tres años tras el tratamiento quirúrgico primario y ningún caso con recurrencia temprana, que equivale a la aparición de la recurrencia de hernia inguinal antes de los tres años, quedando dentro del rango que resalta la revista Universidad y Ciencia de la ciudad de México en su estudio Recurrencia de Hernias Inguinales durante año 2016, que indica dicha aparición tardía se pueda deber a factores propiamente del paciente y no asociados a técnica quirúrgica.

La escala de Campanelli implementada desde el año 1996 ha sido de referencia para clasificación de hernias inguinales recidivantes o recurrentes la cual va desde tipo R1 a R3 respectivamente, según resultados de éste estudio en base a la clasificación se encuentra que hay un predominio de $\mathrm{R} 2$ con un total de $60 \%$ (6) de pacientes, pertenecientes a primera recidiva de hernia inguinal tipo directa y en R1 con un $40 \%$ (1) de pacientes correspondientes a primera recidiva de hernia inguinal tipo indirecta y R3 sin ningún caso pertenecientes a todas las hernias inguinales recidivadas más de una vez.

Tomando en cuenta la clasificación según la escala de Nyhus el 60\% (6) de los pacientes tuvo hernia inguinal recurrente tipo directa y el $40 \%$ (4) de los pacientes correspondes a hernia inguinal recurrente tipo indirecta y ningún paciente con hernia inguinal recurrente se encontró con el tipo combinada. La localización anatómica frecuente que se presentó en pacientes con hernia inguinal recurrente fue lado izquierdo con un total de 7 casos que equivale a un $70 \%$ y en segundo lugar con 3 caso equivalente al $30 \%$ se encuentran lado derecho; sin encontrarse hernias recurrentes bilaterales.

Se observó que de la técnica más utilizada en la primera intervención quirúrgica del total de pacientes atendidos con hernia inguinal que correspondieron a 137 casos se tiene en primer lugar con un $71 \%$ (97) casos la técnica utilizada con dispositivos fue la de Lichtenstein, segundo lugar con un 17\% (23) casos con otras técnicas entre las cuales se encontró tipo Harkins, tercer lugar con un 9\% (13) casos técnica de Bassini que es una de las técnicas estudiadas que han demostrado mayor recurrencia de hernia inguinal según el estudio de la Universidad y Ciencia de la Ciudad de México y por último con un 1\% (2) casos técnica de Fergunson Andrews, 1\% (2) casos encontrado con la técnica de Shouldice. 
En cuanto a la reparación de hernias inguinales recurrentes la técnica más utilizada en la intervención quirúrgica primaria del total de pacientes atendidos con hernia inguinal recurrente que corresponde a 10 pacientes; 40\% (4) pacientes se realizó reparación con técnica de Bassini, en segundo lugar, el 20\% (2) casos con técnica de Ferguson Andrews, tercer lugar 20\% (2) casos con otras técnicas, en cuarto lugar 10\% (1) caso con técnica de Lichtenstein y 10\% (1) caso con técnica Shouldice. Aumentando así la probabilidad que la recurrencia continúe, ya que la única técnica sin tensión de la cual se ha demostrado una reducción del 50 a 75\% de la recurrencia de hernia inguinal es el tipo Lichtenstein; según el estudio reparación abierta de hernia inguinal: una revisión basada en pruebas de la Revista de Clínicas Quirúrgicas de Norteamérica publicada en el año 2008.

Además se logró evidenciar en el presente estudio que la mayoría de hernioplastias efectuadas con un $60 \%$ (6) casos fueron intervenidos de forma electiva y el $40 \%$ (4) casos fueron intervenidos de forma urgente, comparado con el estudio "Comportamiento de las hernias inguinales y su recurrencia en el servicio de cirugía del Hospital Nacional de Chiquimula en el año 2007” en el cual la mayoría siguen siendo de reparación electiva, queda demostrado que el tipo de cirugía no ha influido en la recurrencia de las mismas.

\section{Agradecimientos}

Al Hospital Regional de Zacapa, en especial al Comité de Docencia e Investigación por la aprobación para realizar la investigación. Un agradecimiento especial a los doctores Edvin Danilo Mazariegos Albanés, Graciela Icaza y Carlos Arriola Monasterio, por ser unos excelentes guías y apoyo durante la realización de la investigación.

\section{Referencias bibliógraficas}

Barrera, W. (2015). Manejo de pacientes con hernia inguinal recidivante, Guatemala, USAC. p. 41. Disponible en http:/www.repositorio.usac.edu.gt/4340/1/WENCESLAO \% 20BARRERA\%20 GODOY.pdf

Buitrago, J., Vera, F., Martínez, D., Muñoz, D. \& Sánchez, D. (2014). ¿Intervenimos adecuadamente las hernias inguinales? Revista Colombiana de Cirugía 29:116-122. Disponible en http://www.scielo. org.co/pdf/rcci/v29n2/v29n2a6.pdf

Chinchilla, L. (2007). Comportamiento de las hernias inguinales y su recurrencia en pacientes de cirugía General del Hospital Modular de Chiquimula. Guatemala, USAC-CUNORI.

Palacio, A. (2016). Epidemiología y factores que influyen en la recidiva de pacientes intervenidos por hernia inguinal en una Institución de nivel II de la ciudad de Bogotá, 2005.2014 Universidad Nacional de Colombia. p. 41. Disponible en http://bdigital.unal.edu.co/55766/1/1053771162.2016.pdf 


\section{Sobre la autora}

\section{Juliana Hercilia Díaz Gerónimo}

Médico y Cirujano, egresada del Centro Universitario de Oriente, CUNORI, de la Universidad de San Carlos de Guatemala. En el año 2018 realizó su proyecto de investigación con el título "Recurrencia de hernias inguinales en el Hospital regional de Zacapa”.

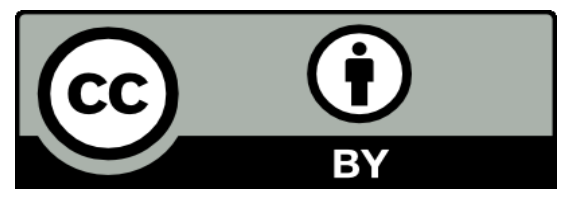

Este texto está protegido por una licencia CreativeCommons 4.0.

Usted es libre para compartir, copiar y redistribuir el material en cualquier medio o formato y adaptar el documento, remezclar, transformar y crear a partir del material para cualquier propósito, incluso comercialmente, siempre que cumpla la condición de atribución: usted debe reconocer el crédito de una obra de manera adecuada, proporcionar un enlace a la licencia, e indicar si se han realizado cambios. Puede hacerlo en cualquier forma razonable, pero no de forma tal que sugiera que tiene el apoyo del licenciante o lo recibe por el uso que hace. 